\title{
Meta-analysis reveals threshold level of rapidly fermentable dietary concentrate that triggers systemic inflammation in cattle
}

\author{
Q. Zebeli, ${ }^{\star} \dagger^{1}$ B. U. Metzler-Zebeli, $† \neq$ and B. N. Ametaj§ \\ *Institute of Animal Nutrition, Department for Farm Animals and Veterinary Public Health, Vetmeduni Vienna, Veterinaerplatz 1 , \\ 1210 Vienna, Austria \\ †Research cluster Animal Gut Health, Vetmeduni Vienna, Veterinaerplatz 1, 1210 Vienna, Austria \\ ¥Clinic for Swine, Department for Farm Animals and Veterinary Public Health, Vetmeduni Vienna, Veterinaerplatz 1, 1210 Vienna, Austria \\ $\S$ Department of Agricultural, Food and Nutritional Science, University of Alberta, Edmonton, AB, Canada T6G 2P5
}

\section{ABSTRACT}

This study examined the extent by which changes in the concentrate level and neutral detergent fiber (NDF) content in the diet as well as the severity of acidotic insult, measured as the duration time of rumen $\mathrm{pH}$ below 6.0 and daily mean rumen $\mathrm{pH}$, and the concentration of endotoxin in the rumen fluid are involved in the development of inflammatory conditions in cattle. A meta-analytical approach accounting for inter- and intraexperimental variation was used to generate prediction models, and data from recent studies were used to parameterize these models. A total of 10 recently conducted experiments with 43 different dietary treatments fulfilled the criteria for inclusion in this study. Diets of all of the experiments included in this meta-analysis were based on rapidly degradable grain sources, such as barley and wheat, and the findings of this study apply only to these kinds of diets. Data indicated that greater levels of concentrate in the diet were associated with increased concentrations of rumen endotoxin $\left(R^{2}=0.27\right)$, plasma haptoglobin $\left(R^{2}=0.19\right)$, and serum amyloid A (SAA) level $\left(\mathrm{R}^{2}=0.46\right)$. Similar correlations, but in opposite directions, were observed between dietary NDF content and rumen endotoxin $\left(\mathrm{R}^{2}=0.39\right)$ and plasma SAA concentrations $\left(\mathrm{R}^{2}=\right.$ 0.22 ). The meta-analysis revealed that the relationships between those variables were not linear. Additionally, the breakpoint model fitted to the data of rumen endotoxin, plasma haptoglobin, and SAA indicated the presence of a threshold level of dietary concentrate and NDF, above which those responses became linear to increasing amounts of concentrate or decreasing contents of NDF in the diet. Also, feeding cattle more than $44.1 \%$ concentrate or less than $39.2 \%$ NDF in the

Received October 23, 2011.

Accepted January 20, 2012.

${ }^{1}$ Corresponding author: Qendrim.Zebeli@vetmeduni.ac.at diet was associated with a linear increase in the risk of systemic inflammation. Low daily mean rumen $\mathrm{pH}\left(\mathrm{R}^{2}\right.$ $=0.38)$ and duration of rumen $\mathrm{pH}<6.0\left(\mathrm{R}^{2}=0.59\right)$ were associated with increased concentrations of endotoxin in the rumen fluid; although those events were not always associated with systemic inflammation. Accordingly, only 15 to $21 \%$ of the overall variation in the responses of SAA was explained by variables of rumen $\mathrm{pH}$, whereas the concentrate level in the diet accounted for $46 \%$ of this variation. In conclusion, data from this study indicated the presence of thresholds of dietary concentrate and NDF levels in the diets based on rapidly fermentable grains beyond which the risk of systemic inflammation in cattle increases linearly.

Key words: meta-analysis, cattle nutrition, rumen acidosis, systemic inflammation

\section{INTRODUCTION}

The bovine rumen is a classical host microbial ecosystem in which a large diversity of microbiota confers important metabolic capabilities to the host. For instance, the microbiota enable the host to thrive on complex dietary carbohydrates that cannot be digested by mammalian enzymes by providing short-chain FA (SCFA) as the major end products of ruminal fermentation. The SCFA are absorbed directly across the stratified squamous epithelium (SSE) of the reticulorumen (Bergman, 1990). Their absorption is instrumental in supplying energy to the host (Bergman, 1990) and lowering the risk of SARA (Penner et al., 2009).

When the rumen symbiotic relationship is disturbed, such as during SARA, important shifts may occur in its microbiome (Tajima et al., 2000; Fernando et al., 2010; Hook et al., 2011) and rumen metabolite profiles (Ametaj et al., 2010b). Mounting evidence suggests that the latter events are accompanied by a rise in the concentration of LPS, a bioactive cell-wall component of all gram-negative bacteria (GNB), commonly known as endotoxin, in the rumen fluid of steers (Nagaraja et al., 1978; Gozho et al., 2005, 2006) and dairy cows (Gozho 
et al., 2007; Emmanuel et al., 2008; Khafipour et al., 2009c).

Other crucial functions of SSE of the reticulorumen are to maintain epithelial tissue barrier integrity while regulating the absorption of nutrients (Baldwin, 1998). The barrier function of the SSE is particularly important in cattle during the long episodes of SARA to prevent translocation of the accumulated LPS or other toxic compounds (Ametaj et al., 2010b) into the systemic circulation. When translocated into the systemic circulation, LPS has been shown to induce the release of a whole variety of acute phase proteins (APP), from liver hepatocytes as part of a general nonspecific inflammatory response (Emmanuel et al., 2008; Khafipour et al., 2009c). Although the inflammatory response is regarded as a protective reaction of the body aiming to re-establish the disturbed homeostasis, in the long term, strong inflammatory states might have implications for animal health (Elsasser et al., 2008; Ametaj et al., 2010a, 2011).

Aspects of the relationships among SARA, rumen endotoxin, and their resulting pathophysiological effects on the rumen and cow's metabolic status have been the focus of intensive research recently (Plaizier et al., 2008; Ametaj et al., 2010a); however, there have been some discrepancies in the results obtained. For example, although changes in the permeability of rumen SSE due to SARA have been evidenced (Emmanuel et al., 2007; Steele et al., 2011), the translocation of endotoxin associated with a systemic inflammation has been reported in some studies (Gozho et al., 2007; Emmanuel et al., 2008; Khafipour et al., 2009c), but not in others (Khafipour et al., 2009a; Iqbal et al., 2010).

Although the exact mechanisms responsible for these discrepancies in the results are not clear yet, several factors such as diet composition (Khafipour et al., 2009a; Iqbal et al., 2010), luminal LPS load (Emmanuel et al., 2007), overgrowth of rumen GNB with certain virulence factors (Khafipour et al., 2011), and adaptive changes occurring in the rumen SSE dependent on duration and severity of SARA challenge (Steele et al., 2009, 2011) have been suggested to play a role. A recent study by Penner et al. (2010) indicated that a mild episode of SARA (time of $\mathrm{pH}<5.8$ of $111 \mathrm{~min}$ ) did not affect ruminal epithelial barrier function, but a rapid and more severe in vitro acidification ( $\mathrm{pH} 5.2)$ increased epithelial permeability, indicating that the severity and duration of SARA might also play a role. Consequently, a need exists to examine whether individual changes in the diet (i.e., concentrate level and NDF content), severity of acidotic insult (i.e., time of $\mathrm{pH}$ below a certain threshold level of SARA and daily mean $\mathrm{pH}$ ), and concentration of rumen endotoxin are involved in the inflammatory responses.
Recently, meta-analytical studies have been used in animal sciences to resolve discrepancies in the literature and to use existing data to quantify multiple relationships (St-Pierre, 2001), hence addressing hypotheses that could not be addressed in one single study. In this meta-analysis, we examined the role of concentrate level and NDF content in the diet, duration of SARA, and concentration of endotoxin in the rumen fluid as potential risk factors of inflammatory responses in cattle.

\section{MATERIALS AND METHODS}

\section{Data Search}

As a first step, a literature search was conducted using public data search generators, such as PubMed, Google Scholar, ScienceDirect, and Scopus, as well as contact with researchers in the field to identify published articles on rumen $\mathrm{pH}$, rumen endotoxin, and inflammatory responses in blood plasma. The search strategy aimed to identify articles that contained specific data on controlled experiments examining the effects of dietary concentrate, either the amount or degradation, or the effects of a specific grain ingredient intervention (e.g., barley processing) on rumen fermentation, LPS, and inflammatory markers. The following key words, in different combinations, were used for our search: rumen LPS, rumen pH, SARA, acute phase proteins, inflammation markers, cattle, cow, steers. The most commonly reported inflammatory biomarkers were identified to be serum amyloid A (SAA) and haptoglobin (Hp), whereas LPS-binding protein (LBP) was reported in fewer studies. Because ruminal acidosis has been viewed as a causal factor of inflammation (Emmanuel et al., 2007), and rumen $\mathrm{pH}$ is often used as the most important indicator of ruminal acidosis and health (Enemark, 2008; Zebeli et al., 2008; Plaizier et al., 2008), particular attention was paid to include representative values of rumen $\mathrm{pH}$ in the meta-analysis. For this, rumen $\mathrm{pH}$ measurements, reported both as daily mean and the time of $\mathrm{pH}<6.0$, were included. Sufficient data were also reported about the concentration of VFA in the rumen fluid. Details on dietary formulation and chemical composition of diets were extracted from the articles. A database was built using these data in an Excel (Microsoft Corp., Redmond, WA) spreadsheet. A list of publications reviewed for the study is provided in Table 1. Indeed, the ranges in the level of concentrate and the amount of NDF in the diets of studies involved in this meta-analysis were extremely wide. For example, concentrate level varied from 0 to $76 \%$ (DM basis) and NDF content from 25 to $64 \%$ (Table 1). Also, the range of the response variables measured differed considerably. Particularly the 
Table 1. Information about animal numbers (n), experimental animals, dietary characteristics, and range values of dietary components as well as of selected measured rumen and plasma variables (minimum - maximum reported values) from studies included in the meta-analysis

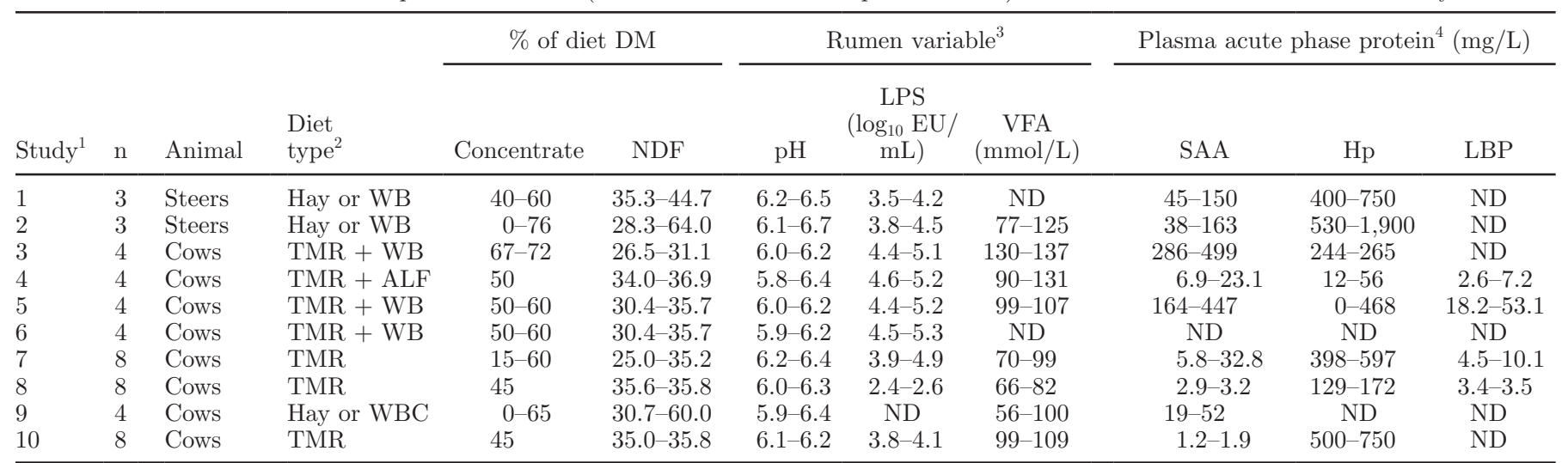

${ }^{1}$ Experiments included in this meta-analysis were 1: Gozho et al. (2005); 2: Gozho et al. (2006); 3: Gozho et al. (2007); 4: Khafipour et al. ( 2009c); 5: Khafipour et al. (2009a); 6: Khafipour et al. (2009b); 7: Zebeli and Ametaj (2009); 8: Iqbal et al. (2009, 2010); 9: Steele et al. (2011); and 10: Iqbal et al. (2012a,b).

${ }^{2} \mathrm{WB}=$ mixture of wheat and barley pellets; $\mathrm{ALF}=$ alfalfa pellets; $\mathrm{WBC}=$ mixture of wheat, barley, and corn.

${ }^{3}$ Rumen $\mathrm{pH}$ reported as a daily mean value; $\mathrm{EU}=$ endotoxin unit.

${ }^{4} \mathrm{SAA}=$ serum amyloid $\mathrm{A} ; \mathrm{Hp}=$ haptoglobin; LBP $=$ LPS-binding protein; $\mathrm{ND}=$ not determined.

concentrations of SAA and Hp in the plasma indicated strong variation within and among the studies included in this meta-analysis (Table 1).

\section{Inclusion Criteria for the Study}

Experiments were included or excluded in this study based on the following criteria. Quality assessment criteria included sufficient data on dietary formulations, rumen $\mathrm{pH}$ measurements throughout the day, data on rumen LPS or plasma APP (or both), clear experimental design, randomization of treatment groups, statistical analysis, and within-study error. Particular emphasis was also placed on the methods of measurement of LPS and inflammatory biomarkers. Lipopolysaccharide content should have been determined by a chromogenic Limulus amoebocyte lysate (LAL) assay. All studies used the LAL test with 96-well microplates with absorbance read at $405 \mathrm{~nm}$ by a microplate spectrophotometer by comparing the LAL concentrations in the rumen fluid supernatant samples with known LPS concentrations contained in the commercially available LAL standards. In all studies, concentrations of SAA and $\mathrm{Hp}$ in the plasma were determined by using commercially available bovine ELISA kits from Tridelta Development Ltd. (Maynooth, Co. Kildare, Ireland). All samples were tested in duplicate and the optical density values in all studies were read on a microplate spectrophotometer at 450 and $630 \mathrm{~nm}$ for SAA and $\mathrm{Hp}$, respectively. Trials were included in the analysis that used dairy cows or steers, and sufficient data were available to determine the effect size (i.e., the number of animals in each treatment group).

\section{Data Extraction and Description of Database}

A total of 10 studies (43 dietary comparisons) met the eligibility criteria for this meta-analysis. The recorded data included authors, journal and year of publication, trial design, type of diet, duration of feeding, number of animals in the treatment groups, concentrate level in the diet, and the content of NDF in the diet. Other information extracted from relevant articles were daily mean rumen $\mathrm{pH}$, duration of rumen $\mathrm{pH}$ below 6.0, VFA concentrations in the rumen fluid, blood SAA, Hp, and $\mathrm{LBP}$ and the respective standard error of each variable. When rumen LPS was reported in $\mathrm{ng} / \mathrm{mL}$, this unit was converted to endotoxin unit $(\mathbf{E U}) / \mathrm{mL}$, assuming a conversion factor of $10 \mathrm{EU}$ for each nanogram of LPS. Rumen LPS was subsequently transformed to $\log _{10}$ EU/ $\mathrm{mL}$. A summary of the response variables considered in this meta-analysis is listed in Table 1.

\section{Data Analysis}

To quantify the responses of animals to predictor variables, all data were subjected to mixed modeling analysis using PROC MIXED (version 9.2; SAS Institute Inc., Cary, NC), and considering the random effect of the study (St-Pierre, 2001), as shown below:

$$
Y_{i j}=\alpha_{0}+\beta_{1} X_{i j}+s_{i}+b_{i} X_{i j}+e_{i j}
$$


where $Y_{i j}=$ the expected outcome for the dependent variable $Y$ observed at level $j(j=2, \ldots, n)$ of the predictor variable $X$ in the study $i$, where $n$ is the number of treatment means in study $i ; \alpha_{0}=$ the overall intercept across all studies (fixed effect); $\beta_{1}=$ the overall regression coefficient of $Y$ on $X$ across all studies (fixed effect); $X_{i j}=$ the value $j$ of continuous variable $X$ in study $i ; s_{i}=$ the random effect of the study $i(i=$ $1, \ldots, 10) ; b_{i}=$ the random effect of study $i$ on the regression coefficient of $Y$ on $X$ in study $i$; and $e_{i j}=$ the unexplained error. Thus, the random effect components of the model include $s_{i}+b_{i} X_{i j}+e_{i j}$, and the distributions are shown below:

$$
e_{i j} \sim \text { iid } N\left(0, \sigma_{\mathrm{e}}^{2}\right) \text { and }\left[\begin{array}{c}
s_{i} \\
b_{i}
\end{array}\right] \sim \text { iid } N\left[\left(\frac{0}{0}\right), \quad \sum\right],
$$

which assumes that $e_{i j}$ is normally distributed with a mean of 0 and constant variance, and that $s_{i}$ and $b_{i}$ are normally distributed, have means of 0 , and $\Sigma$ is their variance-covariance matrix:

$$
\sum=\left[\begin{array}{cc}
\sigma_{s}^{2} & \sigma_{s b} \\
\sigma_{s b} & \sigma_{b}^{2}
\end{array}\right] .
$$

An unstructured variance-covariance structure matrix $(\mathrm{TYPE}=\mathrm{UN})$ was adopted to avoid the positive correlation between the intercepts and slopes, as suggested by St-Pierre (2001). To take the unequal variance among studies into consideration, the dependent variable was weighted by the reciprocal of its squared standard error. When a predictor variable was significant $(P<0.05)$, its squared term was included in the model to test any quadratic relationship. In this case, the variance-covariance matrix was modeled as variance components (TYPE $=\mathrm{VC}$ ), to ensure the convergence.

After a visual inspection of the data using PROC GPLOT (version 9.2; SAS Institute Inc.), responses to predictor variables were modeled using different straight-line breakpoint nonlinear models with the NLMIXED procedure of SAS, as shown below.

$$
Y= \begin{cases}a_{0}+b_{1} \times x, & \text { if } x \leq \pi_{1} \\ a_{0}+b_{1} \times \pi_{1}+b_{2} \times\left(x-\pi_{1}\right) & \text { if } x>\pi_{1}\end{cases}
$$

where $Y$ is the response variable; $a_{0}$ is the general intercept; $b_{1}$ and $b_{2}$ are the slopes for the first and second straight-line segments, respectively; $x$ is the predictor variable, and $\pi_{1}$ is the breakpoint. Estimates and their corresponding $95 \%$ confidence intervals were computed. The confidence interval in this case expresses the likeli- hood for which a range of plausible values of the true estimate lies. Root mean square error (RMSE) and coefficient of determination $\left(\mathrm{R}^{2}\right)$ were subsequently computed and used to evaluate the goodness of fit.

\section{RESULTS}

Data obtained from this meta-analysis indicated that greater levels of concentrate in the diet were associated with increased concentrations of rumen endotoxin (Figure $1 \mathrm{~A})$. The meta-analysis showed that the relationship between these variables was not linear; the breakpoint model fitted to rumen endotoxin data revealed the presence of a threshold level of dietary concentrate, above which the response of rumen endotoxin became responsive to the predictor variable (Figure 1A). It was shown that feeding cattle more than $35 \%$ concentrate in the diet resulted in a linear rise of the concentration of rumen endotoxin (RMSE $=0.55, \mathrm{R}^{2}=0.27, P=$ $0.002)$.

Similar associations were found between the amount of dietary concentrate and responses of plasma $\mathrm{Hp}$ (Figure 1B) and SAA (Figure 1C). Thus, changes in the amount of concentrate in the diet were reflected by changes in the concentration of these inflammation biomarkers in the plasma of cattle. However, as indicated by the breakpoint models that fitted best to the data, the concentration of both variables increased in a linear fashion, particularly when the level of concentrate in the diet exceeded threshold levels of 50 or $44.1 \%$ for $\mathrm{Hp}$ and SAA, respectively. In particular, the equation predicting the response of plasma SAA $\left(\mathrm{R}^{2}=0.46\right)$ to dietary concentrate showed a higher accuracy than equation of the response of plasma $\mathrm{Hp}\left(\mathrm{R}^{2}=0.19\right)$.

Relationships between the content of dietary NDF and rumen endotoxin and plasma SAA are given in Figure 2. Increasing the content of NDF in the diet up to $44.7 \%$ (DM basis) was associated with lowered concentration of rumen endotoxin $\left(\mathrm{R}^{2}=0.39, P<0.001\right.$; Figure 2A). Beyond this threshold of dietary NDF, no further response of rumen endotoxin was observed. Also, the concentration of plasma SAA decreased linearly with increasing the content of dietary NDF up to $39.2 \% \mathrm{NDF}\left(\mathrm{R}^{2}=0.22, P=0.007\right.$; Figure $\left.2 \mathrm{~B}\right)$. According to the equation derived from the association between dietary NDF and plasma SAA, an increase of dietary NDF by $1 \%$ is associated with a decreased concentration of plasma SAA of $9.2 \mathrm{mg} / \mathrm{L}$.

To determine the effects of rumen acidosis on the release of endotoxin in the rumen fluid and activation of systemic inflammation, both data of daily mean rumen $\mathrm{pH}$ and time in which rumen $\mathrm{pH}$ remained $<6.0$ were used in the analysis. Data of daily mean ruminal $\mathrm{pH}$ indicated that low rumen $\mathrm{pH}$ increases the release 

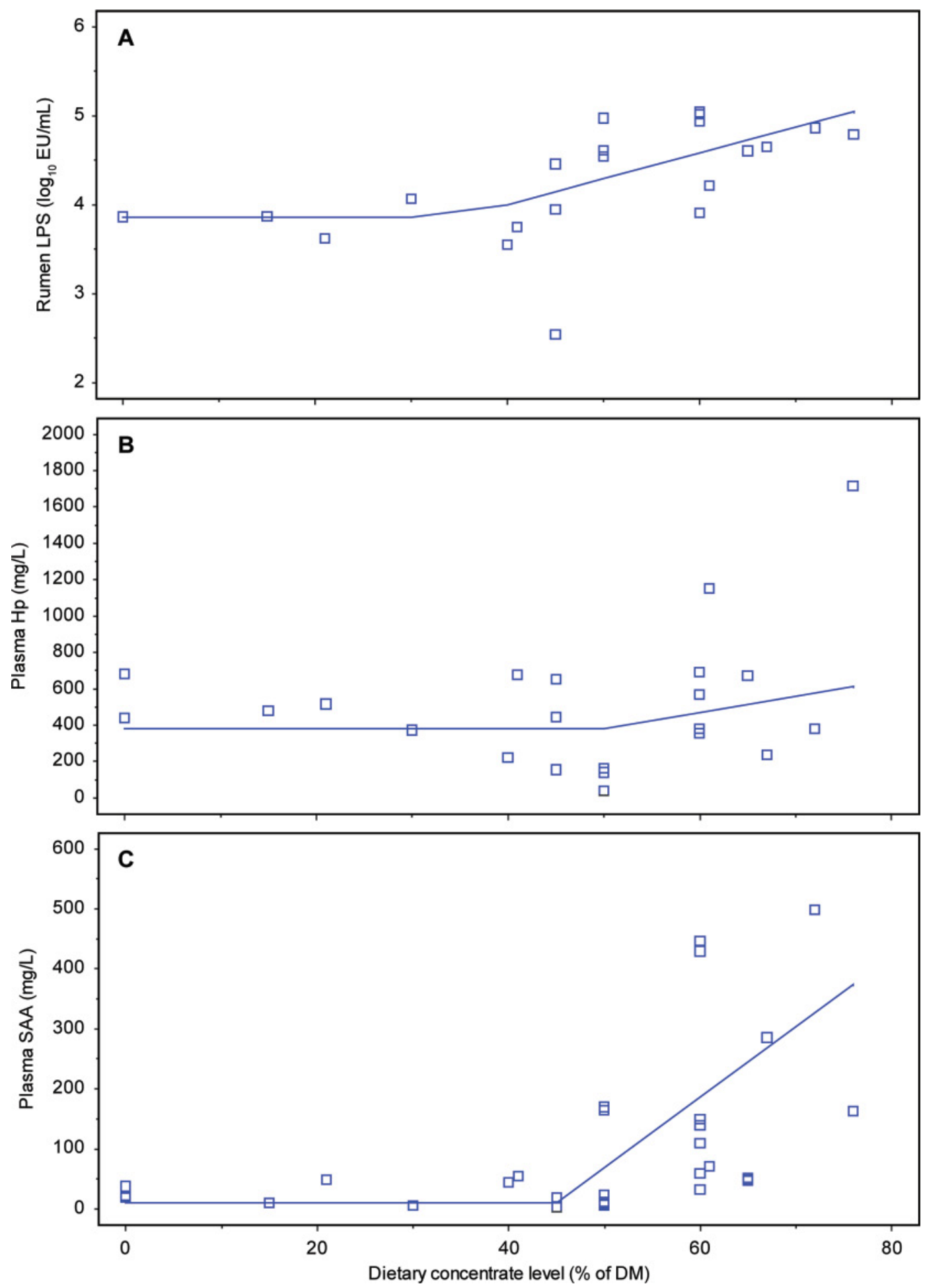

Figure 1. A) Breakpoint model fitted to rumen endotoxin $(y)$ in response to concentrate level in the diet $(x)$ of cattle: $y=\mathrm{a}_{0}+\mathrm{b}_{1} \times x$, if $x>\pi$ $\left[\mathrm{a}_{0}=2.83, \mathrm{~b}_{1}=0.292, \pi\right.$ (breakpoint of $x$ ) $=35.3 \%$ (lower and upper limits of $95 \%$ CI are 14.6 and $56.0 \%$, respectively), asymptotic plateau of $y$ $=3.86 \log _{10}$ endotoxin unit $\left.(\mathrm{EU}) / \mathrm{mL}\right]$; root mean square error $\left.=0.55, \mathrm{R}^{2}=0.27, P=0.002 ; \mathrm{B}\right)$ breakpoint model fitted to plasma haptoglobin (y) in response to concentrate level in the diet $(x)$ of cattle: $y=\mathrm{a}_{0}+\mathrm{b}_{1} \times x$, if $x>\pi\left[\mathrm{a}_{0}=-70.6, \mathrm{~b}_{1}=9.02, \pi\right.$ (breakpoint of $x$ ) $=50 \%$ (lower and upper limits of $95 \%$ CI are 34 and $57 \%$, respectively), asymptotic plateau of $y=380.5 \mathrm{mg} / \mathrm{L}]$; root mean square error $=294.9, \mathrm{R}^{2}=0.19$, $P=0.014 ; \mathrm{C})$ breakpoint model fitted to plasma serum amyloid $\mathrm{A}(y)$ in response to concentrate level in the diet $(x)$ of cattle: $y=\mathrm{a}_{0}+\mathrm{b}_{1} \times x$, if $x>\pi\left[\mathrm{a}_{0}=320.4, \mathrm{~b}_{1}=8.012, \pi\right.$ (breakpoint of $x$ ) $=44.1 \%$ (lower and upper bounds of $95 \%$ CI are 35.7 and $54.3 \%$, respectively), asymptotic plateau of $y=8.34 \mathrm{mg} / \mathrm{L}]$; root mean square error $=96.5, \mathrm{R}^{2}=0.46, P<0.001$. Color version available in the online PDF. 


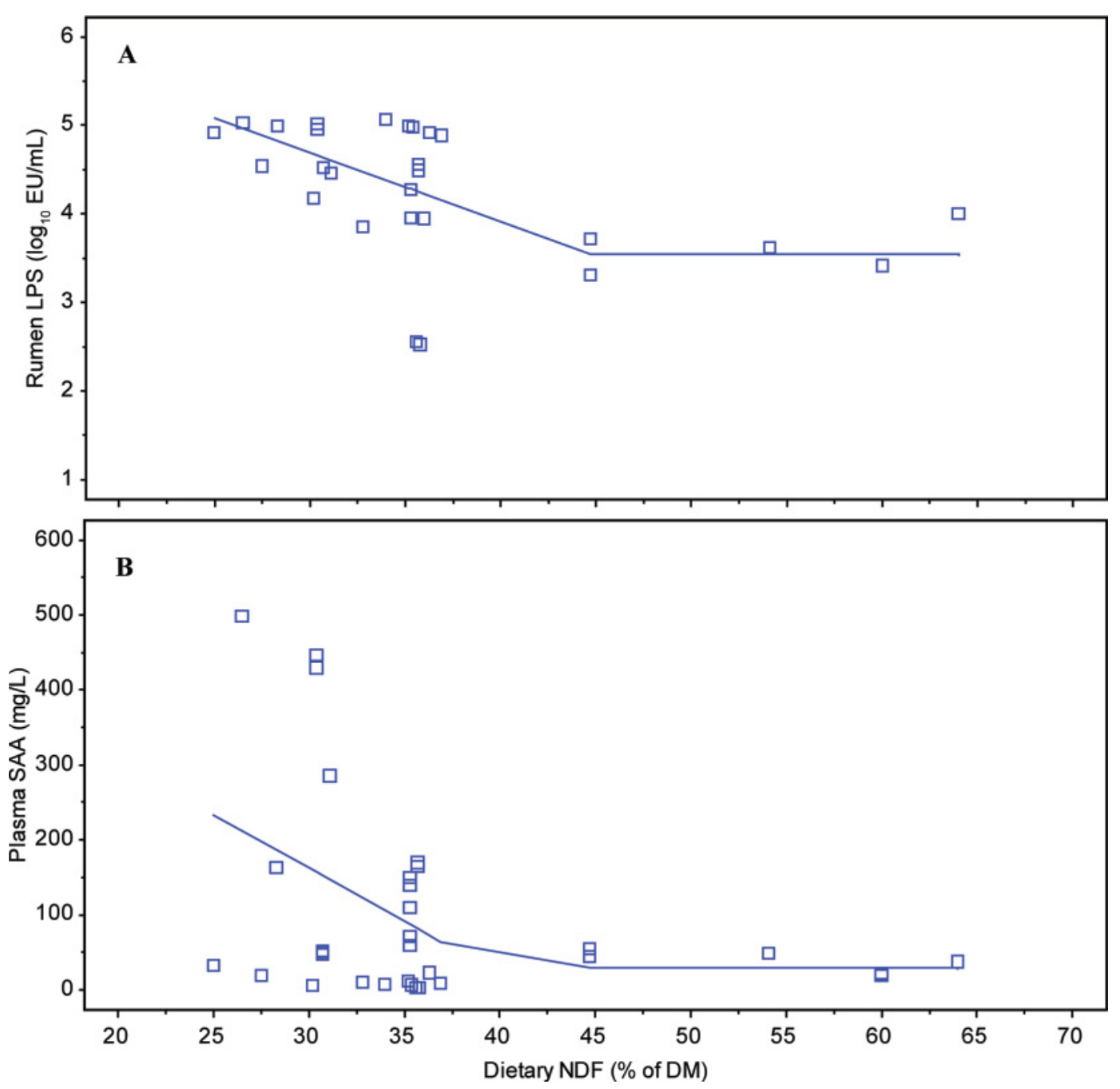

Figure 2. A) Breakpoint model fitted to rumen endotoxin $(y)$ in response to the content of NDF in the diet $(x)$ of cattle: $y=\mathrm{a}_{0}+\mathrm{b}_{1} \times x$, if $x>\pi\left[\mathrm{a}_{0}=7.08, \mathrm{~b}_{1}=-0.077, \pi\right.$ (breakpoint of $\left.x\right)=44.7 \%$ (lower and upper limits of $95 \%$ CI are 35.9 and $53.4 \%$, respectively), asymptotic plateau of $y=3.55 \log _{10}$ endotoxin unit $(\mathrm{EU}) / \mathrm{mL}$; root mean square error $=0.54, \mathrm{R}^{2}=0.39, P<0.001$; B) break-point model fitted to plasma serum amyloid A $(y)$ in response to the content of NDF in the diet $(x)$ of cattle: $y=\mathrm{a}_{0}+\mathrm{b}_{1} \times x$, if $x>\pi\left[\mathrm{a}_{0}=588.9, \mathrm{~b}_{1}=-9.223, \pi\right.$ (breakpoint of $x$ ) $=39.2 \%$ (lower and upper bounds of $95 \%$ CI are 31.6 and $46.9 \%$, respectively), asymptotic plateau of $y=30.1 \mathrm{mg} / \mathrm{L}$ ]; root mean square error $=105.6, \mathrm{R}^{2}=0.22, P=0.007$. Color version available in the online PDF.

of rumen endotoxin $\left(\mathrm{R}^{2}=0.38\right.$; Figure $\left.3 \mathrm{~A}\right)$. Again, a threshold value of daily mean rumen $\mathrm{pH}$ of 6.35 (6.15 and 6.56, lower and upper limits of $95 \% \mathrm{CI}$ ) was detected, under which the concentration of endotoxin in the rumen fluid increased linearly and considerably (from a baseline of $3.92 \log _{10} \mathrm{EU} / \mathrm{mL}$ to $>5 \log _{10} \mathrm{EU} /$ $\mathrm{mL}$ ). A negative relationship existed between daily mean rumen $\mathrm{pH}$ and the concentration of $\mathrm{SAA}$ in the plasma of cattle (Figure 3B). According to the equation generated by this analysis, a decrease of rumen $\mathrm{pH}$ by 0.1 units resulted in $15.6 \mathrm{mg} / \mathrm{L}$ higher SAA concentration in the plasma. In particular, Figure $3 \mathrm{~B}$ shows a certain tendency for SAA to increase in plasma when daily mean rumen $\mathrm{pH}$ decreased below values of 6.3 .
The longer the time during which ruminal $\mathrm{pH}$ remained below 6.0, the higher was the concentration of endotoxin in the rumen fluid (Figure 4A). This analysis indicated that the first $96.5 \mathrm{~min}$ (or up to $317 \mathrm{~min} / \mathrm{d}$ as the upper limit of $95 \%$ CI) do not result in greater release of rumen endotoxin, maintaining a baseline level of $3.69 \log _{10} \mathrm{EU} / \mathrm{mL}$. When time of ruminal $\mathrm{pH}<6.0$ was longer than this latter threshold, an increase in endotoxin concentration of $0.00187 \log _{10} \mathrm{EU} / \mathrm{mL}$ was observed in the rumen fluid for each minute during which rumen $\mathrm{pH}$ remained $<6.0\left(\mathrm{RMSE}=0.43, \mathrm{R}^{2}\right.$ $=0.59$; Figure $4 \mathrm{~A})$. The correlative analysis revealed changes in the response of plasma SAA with increasing time of ruminal $\mathrm{pH}<6.0$ (Figure $4 \mathrm{~B}$ ). The linear model 


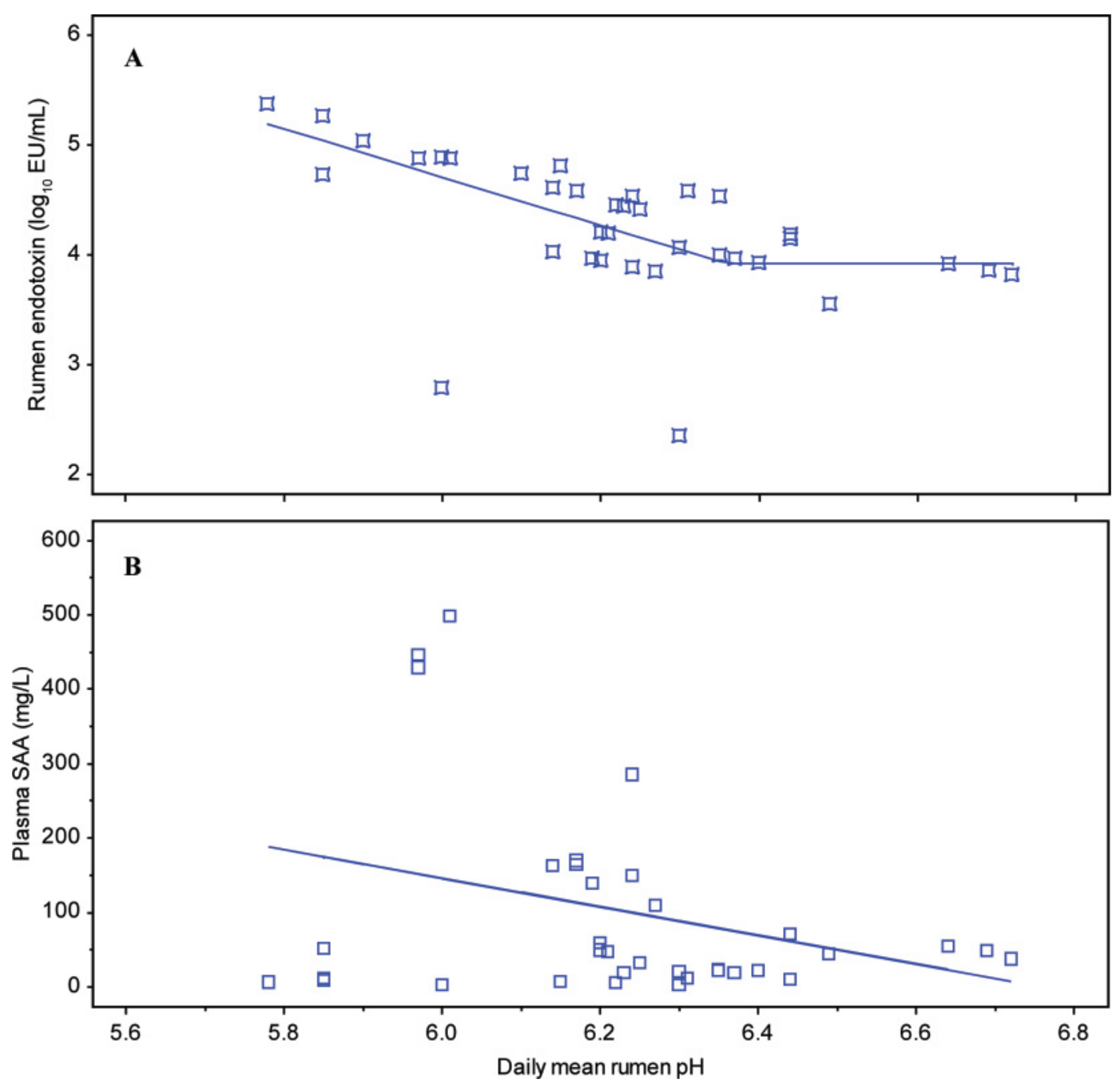

Figure 3. A) Breakpoint model fitted to rumen endotoxin $(y)$ in response to daily mean ruminal $\mathrm{pH}(x)$ in cattle: $y=\mathrm{a}_{0}+\mathrm{b}_{1} \times x$, if $x<\pi$ $\left[\mathrm{a}_{0}=17.84, \mathrm{~b}_{1}=-2.188, \pi\right.$ (breakpoint of $x$ ) $=6.35$ (lower and upper limits of $95 \%$ CI are 6.15 and 6.56 , respectively), asymptotic plateau of $y$ $=3.92 \log _{10}$ endotoxin unit $(\mathrm{EU}) / \mathrm{mL}$; root mean square error $\left.=0.48, \mathrm{R}^{2}=0.38, P<0.001 ; \mathrm{B}\right)$ best-fit linear model of plasma serum amyloid $\mathrm{A}(y)$ in response to daily mean ruminal $\mathrm{pH}(x)$ of cattle: $y=\mathrm{a}_{0}+\mathrm{b}_{1} \times x ; \mathrm{a}_{0}=1,083.15, \mathrm{~b}_{1}=-156.02$, root mean square error $=103.7, \mathrm{R}^{2}=$ $0.15, P=0.043$. Color version available in the online PDF.

fitted to the data of plasma SAA in response to time of ruminal $\mathrm{pH}<6.0$ indicated an increase in plasma SAA of $0.21 \mathrm{mg} / \mathrm{L}$ for each minute when ruminal $\mathrm{pH}<6.0$ $\left(\mathrm{RSME}=111 ; \mathrm{R}^{2}=0.21\right)$.

\section{DISCUSSION}

This study summarizes the most recently published information to determine the role that potential risk factors such as concentrate level and NDF content in the diet, duration and severity of SARA, and the load of endotoxin in the rumen fluid play in the activation of systemic inflammatory responses in cattle. A metaanalytical approach was used to weigh the number of individuals in each experiment, account for the random effect of trial, and the unequal variance among studies due to their experimental differences. Several linear and nonlinear prediction models were generated in this study with their respective breakpoints of the predictor variables, as well as plateau values of the response variables. It should, however, be mentioned that, although the thresholds generated in this study have practical significance in terms of optimizing the effects of predictor variables (i.e., concentrate level and NDF content), the interpretation of the absolute values of these thresholds should be done with caution, because of the relatively small number of observations $(n=43)$ included in this meta-analysis. The use of $95 \%$ confidence interval together with the thresholds might give 


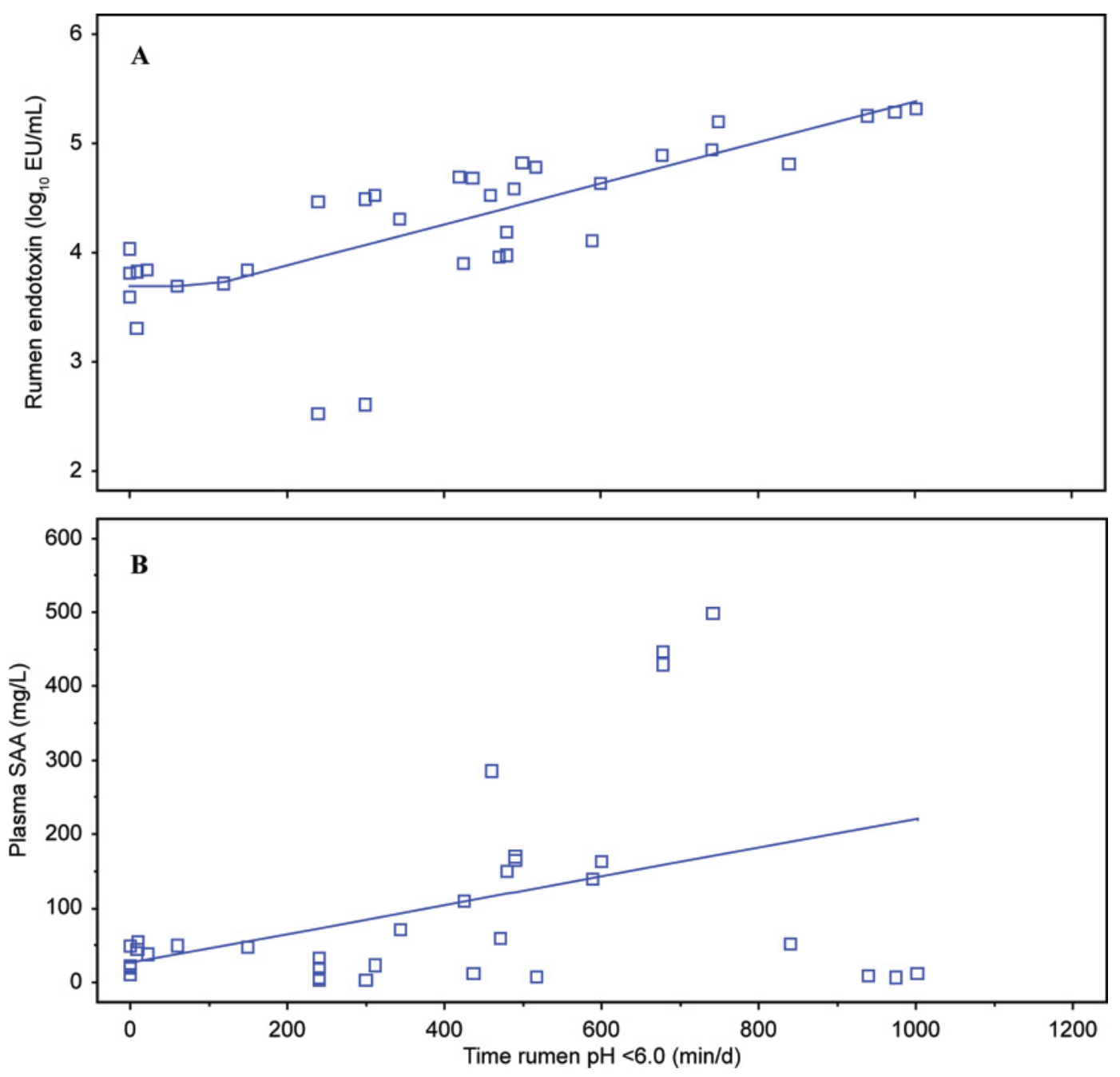

Figure 4. A) Breakpoint model fitted to rumen endotoxin $(y)$ in response to time duration of ruminal $\mathrm{pH}<6.0(x)$ in cattle: $y=\mathrm{a}_{0}+\mathrm{b}_{1}$ $\times x$, if $x>\pi\left[\mathrm{a}_{0}=3.51, \mathrm{~b}_{1}=0.00187, \pi\right.$ (breakpoint of $x$ ) $=96.5 \mathrm{~min} / \mathrm{d}$ (lower and upper limits of $95 \%$ CI are 0 and $317 \mathrm{~min} / \mathrm{d}$, respectively) asymptotic plateau of $y=3.69 \log _{10}$ endotoxin unit $\left.(\mathrm{EU}) / \mathrm{mL}\right]$; root mean square error $=0.43, \mathrm{R}^{2}=0.59, P<0.001$; B) best-fit linear model of plasma serum amyloid $\mathrm{A}(y)$ in response to time of ruminal $\mathrm{pH}<6.0(x)$ in cattle: $y=\mathrm{a}_{0}+\mathrm{b}_{1} \times x ; \mathrm{a}_{0}=14.23, \mathrm{~b}_{1}=0.21$, root mean square error $=111.4, \mathrm{R}^{2}=0.21, P=0.009$. Color version available in the online PDF.

a more accurate measure with regard to the judgment of these values for practical reasons.

The most important outcome of this study was the determination of the extent by which specific changes in the amount of concentrate and the content of NDF in the diet are involved in the release of endotoxin in the rumen, and, most importantly, in the activation of an inflammatory response in cattle. Indeed, identification of threshold levels (i.e., dietary concentrate and NDF levels, ruminal $\mathrm{pH}$, and severity of acidotic insult) is of special interest in terms of characterization of the risk factors for diet-induced inflammation in cattle. Finding an optimal balance between dietary concentrate level and cattle health is a crucial aspect in cattle feeding management schemes. On one hand, cattle diets must contain a high density of ME to support the high energy demands of animals and enhance cost efficiency; on the other hand, this approach can increase the risk of diseases and lower overall profitability (Owens et al., 1998; Stone, 2004). Although substantial research has been undertaken, determination of such a balance is extremely difficult (reviewed by Zebeli et al., 2010).

Interestingly, the threshold of concentrate level ( $>44.1 \%$ of diet $\mathrm{DM}$ ) required to induce inflammation was higher than dietary concentrate threshold $(>35.3 \%$ of diet DM) needed to increase the load of endotoxin in the rumen fluid. The same discrepancy was true for the NDF contents; rumen endotoxin linearly responded to dietary NDF up to a content of $44.7 \%$, whereas the linear increasing response of plasma SAA was noted 
starting from $39.2 \%$ NDF in the diet. Presence of such breakpoint values indicates that the relationships between dietary concentrate level and NDF content with rumen endotoxin and SAA in the plasma are not linear. In addition, different breakpoint values of dietary components (i.e., higher concentrate level or lower NDF content in the diet are needed to increase plasma SAA compared with the amounts needed to increase the level of rumen endotoxin) suggest 2 different modes of action of diets on rumen endotoxin and plasma SAA. Also, these data indicate that cattle may tolerate the increase of a certain level of rumen endotoxin in the rumen fluid before the activation of systemic inflammation occurs. Indeed, the study indicated the presence of a baseline concentration of endotoxin in the rumen fluid. Accordingly, data showed that this baseline was relatively high in cattle $\left(3.6-3.9 \log _{10} \mathrm{EU} / \mathrm{mL}\right)$, indicating that endotoxin is released in cattle rumen fluid independently of the level of concentrate or the severity of rumen acidosis. Because systemic inflammation is initiated when toxic compounds, such as endotoxin, translocate from the gastrointestinal tract to the systemic circulation (Emmanuel et al., 2008; Plaizier et al., 2008), the present findings may indicate that the rumen tolerates a certain endotoxin load $\left(3.6-3.9 \log _{10}\right.$ $\mathrm{EU} / \mathrm{mL}$ ), and probably decrease in ruminal $\mathrm{pH}$, before the rumen SSE becomes impaired and a hepatic acute phase response is initiated.

From all plasma APP tested in this study as inflammation biomarkers, SAA indicated a better accuracy response $\left(R^{2}=0.46\right)$ to the diet, suggesting its appropriateness as a diet-induced inflammation biomarker, for similar studies in cattle, compared with $\mathrm{Hp}\left(\mathrm{R}^{2}=\right.$ 0.19). Despite the fact that APP are synthesized from the same organs (liver and extra hepatic tissues; Ametaj et al., 2011), it is known that their responses in cattle are quite different. This is mostly attributed to their differences in the mode of action as well as in different life times in the plasma. For example, although SAA and LBP have shorter half-life cycles in the circulation than Hp (Gabay and Kushner, 1999), the latter APP is mainly involved in the subacute or chronic inflammatory conditions rather than in acute ones (Horadagoda et al., 1999; Ametaj et al., 2011). Because LBP was reported only in few studies, this variable was not considered in the analysis of this study. The known role of LBP is to facilitate clearance of endotoxin from blood circulation (Schroedl et al., 2001), whereas SAA contributes directly to its neutralization and removal from circulation through liver hepatocytes (Cabana et al., 1999). Haptoglobin is released by the latter cells during bacterial translocation, and its primary function is to bind plasma free hemoglobin, released during hemolysis of red blood cells, and prevent utilization of iron contained in the hemoglobin by translocated bacteria (Wassell, 2000).

In light of today's intensive cattle management systems that encourage inclusion of large amounts of concentrates in the diet to enhance daily energy intake for supporting high milk yields or rapid growth rates, results of this study indicate that feeding of more than $44 \%$ concentrate or less than $39.2 \%$ NDF in the diet linearly increases the risk of systemic inflammation. It should be mentioned that diets included in this meta-analysis included barley or wheat grain, cereal grains rich in easily fermentable carbohydrates. It is well known that diets containing the same amount of NDF or physically effective NDF, but more readily fermentable carbohydrates (e.g., starch) have a higher risk of rumen metabolic disorders (Stone, 2004; Zebeli et al., 2008) and, as shown in this study, greater risk of inflammation. Therefore, the threshold of concentrate (i.e., $44.1 \%)$ or NDF content $(39.2 \%)$ in the diet required for induction of a linear increase of plasma SAA can be viewed as relevant only for these types of diets, and the outcome of this study applies only for diets based on such grain sources. For diets containing grains with slowly degradable starches with a lower capacity to induce fermentation disorders in the rumen, such as corn grain (Zebeli et al., 2008), the threshold of dietary concentrate needed to induce systemic inflammation might be reached at a higher level.

Feeding diets with $>44 \%$ concentrate or $<39 \%$ NDF in cattle, even when diets are based on barley grains, is a very frequent practice in intensive cattle production. Results of this study indicate that this feeding practice increases the risk of systemic inflammation, which is a milder form of inflammation compared with endotoxemia due to intravenous endotoxin injection or during infection with GNB. The latter states are known to be harmful to the host, as they increase the host susceptibility to other diseases and also augment the requirements in energy and nutrients, which may result in lowered efficiency of energy and feed use by the animal (Elsasser et al., 2008). Although data from this study indicate an increased risk of diet-induced inflammation in cattle fed rapidly fermentable concentrates, further research is needed to determine consequences of this kind of inflammation for health and productivity of cattle.

Lowered rumen $\mathrm{pH}$ at SARA levels is often viewed as an indicator of impaired rumen health. Rumen $\mathrm{pH}$ is lowered in response to the rapid accumulation of SCFA produced by microbial fermentation, and when rumen buffering capacity cannot keep pace with the accumulation of these acids during periods of excessive ruminal fermentation (Owens et al., 1998; Stone, 2004). Results of this study showed that low rumen $\mathrm{pH}$, and particu- 
larly more than $96.5 \mathrm{~min} / \mathrm{d}$ during which ruminal $\mathrm{pH}$ was $<6.0$, resulted in linear release of endotoxin in the rumen fluid. This result is not surprising because it is known that the concentration of endotoxin increases in the rumen fluid during the logarithmic growth phase (Hurley, 1995) and massive lysis of the GNB due to SARA conditions (Nagaraja et al., 1978).

Endotoxin is a strong proinflammatory molecule consisting of a lipid region (termed lipid A) attached to a polysaccharide region. The polysaccharide region is composed of 3 separate components: an inner core, an outer core, and the $O$-specific chain or $O$-polysaccharide (Janssens and Beyaert, 2003). It is hypothesized that mechanical disruption of the reticuloruminal SSE barriers during SARA may also open the route of microbial toxins to translocate to the systemic circulation (Plaizier et al., 2008). Indeed, mounting evidence, during recent years, indicates that rumen mucosal barriers are impaired during long episodes of SARA (Emmanuel et al., 2007; Steele et al., 2009; Penner et al., 2010).

However, as shown in this study, the severity of SARA (low rumen $\mathrm{pH}$ and long duration of rumen $\mathrm{pH}$ ) and high concentrations of endotoxin in the rumen fluid are not always reflected in systemic inflammation. This fact is supported by the results of the relationship between rumen acidosis variables and plasma SAA, whereby the predictor variables of rumen $\mathrm{pH}$ explained only 15 to $21 \%$ of the variation in the responses of plasma SAA, indicating that other factors, besides ruminal $\mathrm{pH}$, are important for inducing a systemic inflammation in cattle. Although endotoxin is often used as single marker for the release of toxic compounds during SARA, other toxins, such as biogenic amines, also become more abundant during acidotic insults (Plaizier et al., 2008). For instance, feeding increasing amounts of grain in the diet dramatically raised ruminal concentrations of endotoxin, ethanol, and biogenic amines, such as methylamine and nitrosodimethylamine (Ametaj et al., 2010a). Another indication that other compounds are involved in the impairment of the SSE comes from the study by Khafipour et al. (2009b) where feeding of $50 \%$ grain and alfalfa pellets caused low ruminal $\mathrm{pH}$ and elevated amounts of endotoxin in rumen fluid, but no translocation of endotoxin was observed in their study.

\section{CONCLUSIONS}

Taken together, this study identified thresholds of dietary concentrate level and NDF content in the diets based on rapidly fermentable grains beyond which the inflammatory biomarkers in the plasma such as SAA increased linearly. The study emphasizes the need for developing feeding strategies that help in the mitigation of the effect of high levels of rapidly fermentable con- centrates in the diet on systemic inflammation without lowering the energy and nutrient intake of the animal. Further research is warranted to better understand the effect of feeding rapidly fermentable concentrate on the disruption of the barrier function of the rumen SSE, as well as the inner homeostasis of the animal, and also to determine consequences of diet-induced inflammation on health and productivity in cattle.

\section{REFERENCES}

Ametaj, B. N., A. Hosseini, J. F. Odhiambo, S. Iqbal, S. Sharma, Q. Deng, T. H. Lam, U. Farooq, Q. Zebeli, and S. M. Dunn. 2011. Application of acute phase proteins for monitoring inflammatory states in cattle. In Acute Phase Proteins as Early NonSpecific Biomarkers of Human and Veterinary Diseases. F. Veas, ed. InTech, Rijeka, Croatia. Accessed Dec. 22, 2011. http://www. intechopen.com/articles/show/title/application-of-acute-phaseproteins-for-monitoring-inflammatory-states-in-cattle.

Ametaj, B. N., Q. Zebeli, and S. Iqbal. 2010a. Nutrition, microbiota, and endotoxin-related diseases in dairy cows. R. Bras. Zootec. 39(Suppl. 1):433-444.

Ametaj, B. N., Q. Zebeli, F. Saleem, N. Psychogios, M. J. Lewis, S. M. Dunn, J. Xia, and D. S. Wishart. 2010b. Metabolomics reveals unhealthy alterations in rumen metabolism with increased proportion of cereal grain in the diet of dairy cows. Metabolomics 6:583-594.

Baldwin, R. L. VI. 1998. Use of isolated ruminal epithelial cells in the study of rumen metabolism. J. Nutr. 128:293S-296S.

Bergman, E. N. 1990. Energy contributions of volatile fatty acids from the gastrointestinal tract in various species. Physiol. Rev. 70:567-590.

Cabana, V. G., C. A. Reardon, B. Wei, J. R. Lukens, and G. S. Getz. 1999. SAA-only HDL formed during the acute phase response in apoA-I+/+ and apoA-I-/- mice. J. Lipid Res. 40:1090-1103.

Elsasser, T. H., T. J. Caperna, C.-J. Li, S. Kahl, and J. L. Sartin. 2008. Critical control points in the impact of the proinflammatory immune response on growth and metabolism. J. Anim. Sci. 86:E105-E125.

Emmanuel, D. G. V., S. M. Dunn, and B. N. Ametaj. 2008. Feeding high proportions of barley grain stimulates an inflammatory response in dairy cows. J. Dairy Sci. 91:606-614.

Emmanuel, D. G. V., K. L. Madsen, T. A. Churchill, S. M. Dunn, and B. N. Ametaj. 2007. Acidosis and lipopolysaccharide from Escherichia coli B:055 cause hyperpermeability of rumen and colon tissues. J. Dairy Sci. 90:5552-5557.

Enemark, J. M. D. 2008. The monitoring, prevention and treatment of sub-acute ruminal acidosis (SARA): A review. Vet. J. 176:32-43.

Fernando, S. C., H. T. Purvis II, F. Z. Najar, L. O. Sukharnikov, C. R. Krehbiel, T. G. Nagaraja, B. A. Roe, and U. DeSilva. 2010. Rumen microbial population dynamics during adaptation to a highgrain diet. Appl. Environ. Microbiol. 76:7482-7490.

Gabay, C., and I. Kushner. 1999. Acute-phase proteins and other systemic response to inflammation. N. Engl. J. Med. 340:448-454.

Gozho, G. N., D. O. Krause, and J. C. Plaizier. 2006. Rumen lipopolysaccharide and inflammation during grain adaptation and subacute ruminal acidosis in steers. J. Dairy Sci. 89:4404-4413.

Gozho, G. N., D. O. Krause, and J. C. Plaizier. 2007. Ruminal lipopolysaccharide concentration and inflammatory response during grain-induced subacute ruminal acidosis in dairy cows. J. Dairy Sci. 90:856-866.

Gozho, G. N., J. C. Plaizier, D. O. Krause, A. D. Kennedy, and K. M. Wittenberg. 2005. Subacute ruminal acidosis induces ruminal lipopolysaccharide endotoxin release and triggers an inflammatory response. J. Dairy Sci. 88:1399-1403.

Hook, S. E., M. A. Steele, K. S. Northwood, J. Dijkstra, J. France, A.-D. G. Wright, and B. W. McBride. 2011. Impact of subacute ruminal acidosis (SARA) adaptation and recovery on the densi- 
ty and diversity of bacteria in the rumen of dairy cows. FEMS Microbiol. Ecol. 78:275-284. http://dx.doi.org/10.1111/j.15746941.2011.01154.x.

Horadagoda, N. U., K. M. G. Knox, H. A. Gibbs, S. W. J. Reid, A. Horadagoda, S. E. R. Edwards, and P. D. Eckersall. 1999. Acute phase proteins in cattle: Discrimination between acute and chronic inflammation. Vet. Rec. 144:437-441.

Hurley, J. C. 1995. Endotoxemia: Methods of detection and clinical correlates. Clin. Microbiol. Rev. 8:268-292.

Iqbal, S., S. J. Terrill, Q. Zebeli, A. Mazzolari, S. M. Dunn, W. Z. Yang, and B. N. Ametaj. 2012a. Treating barley grain with lactic acid and heat prevented sub-acute ruminal acidosis and increased milk fat content in dairy cows. Anim. Feed Sci. Technol. 172:141-149.

Iqbal, S., Q. Zebeli, A. Mazzolari, G. Bertoni, S. M. Dunn, W. Z. Yang, and B. N. Ametaj. 2009. Feeding barley grain steeped in lactic acid modulates rumen fermentation patterns and increases milk fat content in dairy cows. J. Dairy Sci. 92:6023-6032.

Iqbal, S., Q. Zebeli, A. Mazzolari, S. M. Dunn, and B. N. Ametaj. 2010. Feeding barley grain treated with lactic acid modulates energy status and innate immunity in dairy cows. J. Dairy Sci. 93:5147-5156.

Iqbal, S., Q. Zebeli, A. Mazzolari, S. M. Dunn, and B. N. Ametaj. 2012b. Barley grain-based diet treated with lactic acid and heat modulated plasma metabolites and acute phase response in dairy cows. J. Anim. Sci. http://dx.doi.org/http://dx.doi.org/10.2527/ jas.2011-3983.

Janssens, S., and R. Beyaert. 2003. Role of toll-like receptors in pathogen recognition. Clin. Microbiol. Rev. 16:637-646.

Khafipour, E., D. O. Krause, and J. C. Plaizier. 2009a. A grain-based subacute ruminal acidosis challenge causes translocation of lipopolysaccharide and triggers inflammation. J. Dairy Sci. 92:10601070.

Khafipour, E., D. O. Krause, and J. C. Plaizier. 2009b. Alfalfa pellet induced subacute ruminal acidosis in dairy cows increases bacterial endotoxin in the rumen without causing inflammation. J. Dairy Sci. 92:1712-1724

Khafipour, E., S. Li, J. C. Plaizier, and D. O. Krause. 2009c. Rumen microbiome composition determined using two nutritional models of subacute ruminal acidosis. Appl. Environ. Microbiol. 75:7115-7124

Khafipour, E., J. C. Plaizier, P. C. Aikman, and D. O. Krause. 2011. Population structure of rumen Escherichia coli associated with subacute ruminal acidosis (SARA) in dairy cattle. J. Dairy Sci. 94:351-360.

Nagaraja, T. G., E. E. Bartley, L. R. Fina, H. D. Anthony, and R. M. Bechtle. 1978. Evidence of endotoxins in the rumen bacteria of cattle fed hay or grain. J. Anim. Sci. 47:226-234.
Owens, F. N., D. S. Secrist, W. J. Hill, and D. R. Gill. 1998. Acidosis in cattle: A review . J. Anim. Sci. 76:275-286.

Penner, G. B., J. R. Aschenbach, G. Gäbel, R. Rackwitz, and M. Oba. 2009. Epithelial capacity for apical uptake of short chain fatty acids is a key determinant for intraruminal $\mathrm{pH}$ and the susceptibility to subacute ruminal acidosis in sheep. J. Nutr. 139:1714-1720.

Penner, G. B., M. Oba, G. Gäbel, and J. R. Aschenbach. 2010. A single mild episode of subacute ruminal acidosis does not affect ruminal barrier function in the short term. J. Dairy Sci. 93:4838-4845.

Plaizier, J. C., D. O. Krause, G. N. Gozho, and B. W. McBride. 2008. Subacute ruminal acidosis in dairy cows: The physiological causes, incidence and consequences. Vet. J. 176:21-31.

Schroedl, W., B. Fuerll, P. Reinhold, M. Krueger, and C. Schuett. 2001. A novel acute phase marker in cattle: Lipopolysaccharide binding protein (LBP). J. Endotoxin Res. 7:49-52.

St-Pierre, N. R. 2001. Invited review: Integrating quantitative findings from multiple studies using mixed model methodology. J. Dairy Sci. 84:741-755.

Steele, M. A., O. AlZahal, S. E. Hook, J. Croom, and B. W. McBride. 2009. Ruminal acidosis and the rapid onset of ruminal parakeratosis in a mature dairy cow: A case report. Acta Vet. Scand. 51:39.

Steele, M. A., J. Croom, M. Kahler, O. AlZahal, S. E. Hook, K. Plaizier, and B. W. McBride. 2011. Bovine rumen epithelium undergoes rapid structural adaptations during grain-induced subacute ruminal acidosis. Am. J. Physiol. Regul. Integr. Comp. Physiol. 300:R1515-R1523. http://dx.doi.org/10.1152/ajpregu.00120.2010.

Stone, W. C. 2004. Nutritional approaches to minimize subacute ruminal acidosis and laminitis in dairy cattle. J. Dairy Sci. 87(E.Suppl.):E12-E26.

Tajima, K., S. Arai, K. Ogata, T. Nagamine, H. Matsui, M. Nakamura, R. I. Aminov, and Y. Benno. 2000. Rumen bacterial community transition during adaptation to high-grain diet. Anaerobe 6:273-284

Wassell, J. 2000. Haptoglobin: Function and polymorphism. Clin. Lab. 46:547-552.

Zebeli, Q., and B. N. Ametaj. 2009. Relationships between rumen lipopolysaccharide and mediators of inflammatory response with milk fat production and efficiency in dairy cows. J. Dairy Sci. 92:3800-3809

Zebeli, Q., J. Dijkstra, M. Tafaj, H. Steingass, B. N. Ametaj, and W. Drochner. 2008. Modeling the adequacy of dietary fiber in dairy cows based on the responses of ruminal $\mathrm{pH}$ and milk fat production to composition of the diet. J. Dairy Sci. 91:2046-2066.

Zebeli, Q., D. Mansmann, H. Steingass, and B. N. Ametaj. 2010. Balancing diets for physically effective fibre and ruminally degradable starch: A key to lower the risk of sub-acute rumen acidosis and improve productivity of dairy cattle. Livest. Sci. 127:1-10. 